\title{
Ablação Histeroscópica do Endométrio no Tratamento da Menorragia - Seguimento de 200 Casos
}

\author{
Hysteroscopic Endometrial Ablation for the Treatment \\ of Menorrhagia - Follow-up of 200 Cases
}

Francesco Viscomi, João Alfredo Martins, Marilice Dall'Aglio Pastore

\begin{abstract}
RESUMO
Objetivo: avaliar a eficácia da ablação histeroscópica do endométrio no tratamento cirúrgico da menorragia.

Métodos: foram realizadas 200 ablações histeroscópicas do endométrio no periodo de abril de 1991 a abril de 1999 em pacientes de clinica privada dos autores. Todas as pacientes apresentavam sangramento uterino anormal de causa benigna rebelde ao tratamento clinico. $O$ procedimento foi realizado em ambiente hospitalar sob anestesia de bloqueio (raqui ou peridural). Nos primeiros quatro anos utilizou-se a glicina 1,5\% como meio de distensão da cavidade uterina e nos últimos quatro anos o manitol 3\%. O seguimento variou de 8 meses a 8 anos.

Resultados: das 200 pacientes, 180 (90\%) mostraram-se satisfeitas com o procedimento, relatando diminuição acentuada do fluxo menstrual. A análise histológica do endométrio após ressecção histeroscópica revelou atividade proliferativa em 104 pacientes (52\%), e secretora em 34 (17\%). A hiperplasia endometrial esteve presente em 20 pacientes (10\%). As pacientes que se submeteram à ressecção apresentaram a cavidade uterina diminuida $e$ formação de sinéquias fibrosas, ao passo que nas pacientes que foram submetidas à destruição endometrial a cavidade mostrou-se diminuida porém sem formação de sinéquias. Duas pacientes apresentaram quadro de hipervolemia moderada durante o procedimento, que respondeu bem ao tratamento clínico. Ocorreu um episódio de perfuração uterina durante a fase de dilatação e cinco casos de hematômetra foram registrados.

Conclusões: A ablação histeroscópica do endométrio é um procedimento seguro e eficaz em pacientes selecionadas no tratamento da menorragia de causa benigna rebelde a tratamento clinico.
\end{abstract}

PALAVRAS-CHAVE: Sangramento uterino disfuncional. Endométrio: investigação. Ressecção do endométrio.

\section{Introdução}

Mais de 600.000 histerectomias são realizadas anualmente nos EUA, fazendo com que seja o procedimento cirúrgico ablativo mais comum em Ginecologia $^{1}$. Em torno de 20 a $25 \%$ destes casos

Trabalho realizado no Women's Medical Center - São Paulo Correspondência:

Francesco Visconi

Av. Eusébio Matoso, 196

05423-000 - São Paulo - São Paulo

Telefax: (11) 3819-0577

e-mail: fviscomi@womens.com.br trata-se de sangramento uterino anormal de causa benigna, rebeldes a tratamento clínico, sem patologia uterina. Até há poucos anos as mulheres que não respondessem a tratamento clínico hormonal estavam limitadas a optar por submeter-se à histerectomia ou continuar com seus fluxos menstruais abundantes. A partir da identificação de que a sindrome de Asherman, que é a presença de sinéquias dentro da cavidade uterina, ocasiona amenorréia ou oligomenorréia, qualquer técnica capaz de produzir uma síndrome de Asherman poderia parar o fluxo menstrual aumentado destas pacientes. Surgiram várias técnicas 
químicas e físicas de destruição endometrial, porém a maioria sem sucesso. Com a utilização de laser em 1979, a destruição de endométrio começou a ser realizada com sucesso ${ }^{2}$. DeCherney e Polan $^{3}$ destruíram o endométrio com sucesso, causando amenorréia com a utilização de corrente elétrica de alta freqüência por via histeroscópica. Vancaillie $^{4}$ e Townsend et al. ${ }^{5}$, usaram o eletrodo em roller ball para facilitar a ablação endometrial eletrocirúrgica. Em 1978 Amin e Neuwirth ${ }^{6}$, usando o ressectoscópio urológico para ressecar mioma submucoso, em paciente com sangramento uterino anormal, descreveram a ressecção endometrial transcervical com alça elétrica em semicírculo. $\mathrm{Hamou}^{7}$ desenvolveu a ressecção parcial do endométrio e a utilização de líquidos de baixa viscosidade (glicina 1,5\%) como meio de distensão da cavidade uterina. Atualmente existem outras técnicas de ablação endometrial como radiofreqüência ${ }^{8}$ e, mais recentemente, Neuwirth et al. ${ }^{9}$ relataram o uso de água aquecida dentro de um balão intra-uterino para destruir o endométrio.

O objetivo deste estudo foi avaliar os resultados da ablação histeroscópica do endométrio utilizando-se o ressectoscópio em 200 pacientes.

\section{Pacientes e Métodos}

De abril de 1991 a abril de 1999, 200 pacientes pertencentes à clinica privada dos autores, portadoras de sangramento uterino anormal referido como menorragia rebelde a tratamento clinico foram submetidas à ablação histeroscópica de endométrio. A média de idade das pacientes foi de 40 anos, variando de 31 a 52 anos. A paridade destas pacientes variou de 0 a 4 . Trinta (15\%) pacientes eram nuliparas. A menorragia foi considerada causa de anemia $(\mathrm{Hb}<10,0 \mathrm{~g} / \mathrm{dL})$ em 58 pacientes $(29 \%)$. Além do exame pélvico e de colpocitologia oncótica, todas as pacientes foram submetidas à ultra-sonografia endovaginal e histeroscopia diagnóstica com biópsia de endométrio. A histeroscopia diagnóstica com biópsia de endométrio foi realizada como avaliação pré-operatória e para exclusão de hiperplasias atípicas e adenocarcinoma de endométrio. Os achados histeroscópicos pré-ablação estão descritos na Tabela 1. Pacientes que apresentavam úteros maiores que o correspondente a 12 semanas foram excluídas deste estudo, assim como suspeita de adenomiose e endometriose representaram contra-indicação para este procedimento.

Os análogos agonistas de GnRH (acetato de goserelina) foram utilizados como medicação préoperatória nos casos de anemia (58 - 29\%), com o objetivo de melhorar o déficit hemático, e nos 14 casos $(7 \%)$ de hiperplasia endometrial, para diminuir a espessura do endométrio e facilitar o procedimento cirúrgico. Os análogos do GnRH foram utilizados em 72 pacientes (Grupo I) 3 meses antes do procedimento (2 ampolas). Em 128 pacientes não se utilizou droga no pré-operatório e o procedimento foi realizado na $1^{\mathrm{a}}$ fase do ciclo menstrual, em torno do $8^{\circ}$ dia (Grupo II). Todas as pacientes foram submetidas a anestesia de bloqueio (raqui ou peridural). O instrumental utilizado foi o ressectoscópio 26F, Karl Storz, com alças elétricas em semicírculo e roller ball ligados a fonte elétrica de alta freqüência, com uma potencia de corte e coagulação em torno de 90 watts. O meio de distensão utilizado nos primeiros 4 anos foi a glicina $1,5 \%$ e nos últimos 4 anos o manitol 3\%. A distensão da cavidade uterina foi obtida por pressão hidrostática e pressão manual durante os primeiros 2 anos. Nos últimos 6 anos empregamos o distensor eletrônico Endomat (Karl Storz) que controla automaticamente a pressão, pelo fluxo de líquido.

Tabela 1 - Achados histeroscópicos prévios à ablação histeroscópica do endométrio.

\begin{tabular}{lr}
\hline Achados & $\mathbf{n}$ \\
\hline Cavidade normal & 112 \\
Mioma submucoso & 36 \\
Pólipo endometrial & 28 \\
Hiperplasia endometrial de baixo risco & 14 \\
Mioma submucoso e pólipo endometrial & 10 \\
Total & 200 \\
\hline
\end{tabular}

Em 180 pacientes utilizou-se a técnica de ressecção endometrial com a alça em semicírculo, enviando-se o material endometrial retirado para avaliação histológica. Nas restantes 20 pacientes foi realizada a técnica de destruição do endométrio utilizando-se a alça elétrica tipo roller ball. Ambas as técnicas tiveram como limite inferior de ressecção ou destruição o orifício interno, preservando-se o endométrio do istmo. Após dilatação cervical com vela de Hegar até No. 10, o ressectoscópio foi introduzido dentro da cavidade uterina até o fundo uterino. Com movimento de dentro para fora iniciou-se a ressecção ou a destruição do endométrio. O procedimento iniciou-se em todos os casos na parede posterior, a seguir parede lateral direita, parede anterior e por fim parede lateral esquerda. Nos casos de ressecção endometrial as regiões dos óstios tubáricos e fundo uterino não foram ressecados por risco de per- 
furação e dificuldades anatômicas em relação à alça em semicírculo, dando-se preferência para o uso de roller ball e destruição do endométrio destes locais. Nos casos de ressecção endometrial, ao final do procedimento utilizou-se a alça em roller ball para coagulação do leito sangrante.

Todas as pacientes foram submetidas a histeroscopia diagnóstica entre 40 e 50 dias após o procedimento, com objetivo de se lisar sinéquias frouxas que porventura tivessem se formado no trajeto da cavidade uterina, para evitar hematometras. Os cinco casos $(2,5 \%)$ de hematometra ocorreram após 1 ano do procedimento. Dos cinco casos, quatro foram submetidos a histerectomia laparoscópica supracervical e um caso a correção por via histeroscópica, realizando-se lise de sinéquias. Nos 20 casos em que houve falha do procedimento as pacientes foram submetidas a histerectomia e todas apresentaram diagnóstico anatomopatológico de adenomiose. Para análise comparativa entre os grupos utilizou-se o teste de Mann-Whitney na avaliação das médias e desvios-padrão.

\section{Resultados}

O tempo cirúrgico médio foi de $54,7 \mathrm{minu}-$ tos (variando de 25 a 90 minutos) no grupo de pacientes que não tomou drogas no pré-operatório para preparação do endométrio, e de 18,8 minutos (variando de 12 a 30 minutos) no grupo que tomou droga no pré-operatório. O volume de líquido utilizado como meio de distensão também variou entre os grupos de pacientes que usaram ou não droga no pré-operatório. Em relação ao volume infundido, no grupo de pacientes que usou análogos de GnRH infundiram-se 4,00 L, ao passo que no grupo de pacientes que não fez uso da droga infundiram-se em média $6,59 \mathrm{~L}$ de meio de distensão. $\mathrm{O}$ volume residual, que significa a diferença do líquido utilizado na infusão com o líquido recolhido ao final da cirurgia foi de $0,57 \mathrm{~L}$ no grupo I e 1,07 L no grupo II.

Houve uma diferença significativa entre as variáveis avaliadas: tempo de cirurgia, volume infundido e volume residual. O Grupo I, no qual as pacientes fizeram uso de análogos de $\mathrm{GnRH}$, apresentou valores significativamente menores do que os do Grupo II, que não fizeram uso de análogos de GnRH (Tabela 2).

Duas pacientes apresentaram, como complicações, quadro de hipervolemia moderada, que foi tratada clinicamente, sem intercorrências posteriores. Em 5 pacientes houve um sangramento intra-operatório intenso, sendo colocada uma sonda de Foley intra-uterina com balão distendido até $10 \mathrm{~mL}$ de soro fisiológico. A sonda de Foley permaneceu por $3 \mathrm{~h}$ e após sua retirada houve diminuição significativa do sangramento.

Tabela 2 - Comparação entre os grupos I e || das variáveis tempo de cirurgia, volume infundido e volume residual.

\begin{tabular}{|c|c|c|c|c|c|c|c|}
\hline Grupo & $n$ & média & DP & Mediana & mínimo & Máximo & $p^{*}$ \\
\hline \multicolumn{8}{|c|}{ Tempo de Cirurgia } \\
\hline I & 72 & 18,8 & 4,9 & 18,0 & 12,0 & 30,0 & 0,0001 \\
\hline ॥ & 128 & 54,4 & 20,1 & 52,0 & 25,0 & 90,0 & \\
\hline \multicolumn{8}{|c|}{ Volume Infundido } \\
\hline I & 72 & 4,0 & 1,1 & 4,0 & 2,0 & 6,0 & 0,0001 \\
\hline$\|$ & 128 & 6,6 & 2,1 & 7,0 & 3,0 & 10,0 & \\
\hline \multicolumn{8}{|c|}{ Volume residual } \\
\hline | & 72 & 0,6 & 0,2 & 0,6 & 0,2 & 1,0 & 0,0001 \\
\hline II & 128 & 1,1 & 0,5 & 0,9 & 0,2 & 1,9 & \\
\hline
\end{tabular}

* Teste de Mann-Whitney

Na Tabela 3 são apresentados os resultados da análise histológica do endométrio obtido pela ressecção histeroscópica. Observa-se que foi encontrada atividade proliferativa em 104 pacientes (52\%) e secretora em 34 (17\%). A hiperplasia endometrial esteve presente em 20 pacientes $(10 \%)$.

Em relação a outros achados anatomopato- lógicos encontrados após a ablação histeroscópica do endométrio, houve a confirmação dos achados na avaliação pré-operatória realizada por histeroscopia diagnóstica. Os 20 casos de pacientes que foram submetidas a histerectomia por falha da ablação estão incluídos nos 32 casos de adenomiose (Tabela 4). 
Tabela 3 - Análise histológica do endométrio obtido durante a ablação histeroscópica do endométrio.

\begin{tabular}{lc}
\hline Diagnóstico histológico & $\mathbf{n}$ \\
\hline Endométrio proliferativo & 104 \\
Endométrio secretor & 34 \\
Endométrio misto & 22 \\
Endométrio atrófico & 20 \\
Hiperplasia glandular simples & 12 \\
Hiperplasia glandular cística & 8 \\
Total & 200 \\
\hline
\end{tabular}

Tabela 4 - Achados histológicos associados, no material obtido com a ablação histeroscópica de endométrio.

\begin{tabular}{lc}
\hline Diagnóstico histológico & $\mathbf{n}$ \\
\hline Adenomiose & 32 \\
Mioma submucoso & 42 \\
Pólipo endometrial & 32 \\
\hline
\end{tabular}

Em relação à melhora da sintomatologia, das 200 pacientes submetidas à ablação histeroscópica do endométrio, 180 mostraram-se satisfeitas com o procedimento, relatando diminuição significativa do fluxo menstrual, após um seguimento que variou de 8 a 56 meses (Tabela 5).

Tabela 5 - Padrão menstrual após a ablação histeroscópica do endométrio.

\begin{tabular}{lr}
\hline Diagnóstico histológico & $\mathbf{n}$ \\
\hline Hipomenorréia & 102 \\
Amenorréia & 42 \\
Retorno ao fluxo normal & 36 \\
Inalterado & 20 \\
Total & 200 \\
\hline
\end{tabular}

Em relação à reavaliação pós-ablação, notouse diferença no aspecto da cavidade uterina nas pacientes que foram submetidas à ressecção quando comparadas às que se submeteram à destruição endometrial com roller ball. As pacientes que se submeteram à ressecção apresentaram a cavidade uterina diminuída e formação de sinéquias fibrosas, ao passo que nas pacientes que foram submetidas à destruição endometrial a cavidade mostrou-se diminuída, porém sem formação de sinéquias.

\section{Discussão}

A ablação do endométrio é realizada por ressecção ou destruição, incluindo-se a camada basal e uma porção superficial do miométrio até uma profundidade aproximadamente de $2 \mathrm{~mm}$. A energia elétrica com pulsos de alta freqüência permite a obtenção de material para exame histológico nos casos de ressecção.

O preparo pré-operatório do endométrio com o uso de análogos de GnRH tem sido recomendado por vários autores com o objetivo de facilitar o procedimento $^{10}$. Além de se ter uma espessura menor do endométrio, o sangramento intra-operatório também é menor, diminuindo desta maneira o tempo cirúrgico e utilizando-se um menor volume de líquido distensor, o que faz diminuir as chances de hipervolemia. O uso de análogos de GnRH também se mostra importante em pacientes que apresentam quadro de anemia causada pela menorragia.

A remoção de tecido para análise histológica parece ser a maior vantagem da ressecção endometrial. Este fato não invalida a destruição endometrial, pois admite-se que todas as pacientes que são submetidas à ablação devem necessariamente ser submetidas à histeroscopia diagnóstica prévia com biópsia de endométrio. $\mathrm{O}$ roller ball é um tipo de eletrodo que pode ser usado para destruição do endométrio, tornando o procedimento mais rápido e apresentando pequena penetração para o miométrio.

As complicações como hipervolemia e sangramento aumentado durante o procedimento foram tratadas clinicamente sem intercorrências. Os resultados desta experiência com 200 casos mostrou uma melhora de $90 \%$ da sintomatologia hemorrágica. Estes resultados são similares aos descritos na literatura ${ }^{10-14}$.

Um certo grau de adenomiose foi encontrado em 32 pacientes no material de ablação do endométrio, o que leva a questionar se a adenomiose está relacionada com a falha do método e desta maneira as pacientes portadoras de adenomiose estariam excluídas desta opção terapêutica. Destas 32 pacientes, 20 tiveram seu quadro inalterado, sendo submetidas à histerectomia, e todas apresentaram diagnóstico anatomopatológico de adenomiose. Talvez isto seja verdadeiro no caso de adenomiose grave, porém a questão permanece incerta na literatura.

A ablação de endométrio é um procedimento de hospital-dia, com retorno das pacientes às suas atividades fisicas e profissionais em média dentro de 1 a 2 semanas.

Pode-se concluir que a ablação histeroscó- 
pica de endométrio parece ser mais uma alternativa na abordagem da menorragia em pacientes selecionadas. Esta experiência com 200 casos mostrou que a ablação histeroscópica de endométrio é um procedimento seguro, com bons resultados para um grande número de pacientes com queixa de sangramento uterino anormal de causa benigna e rebelde ao tratamento clínico.

\section{SUMMARY}

Purpose: the aim of the present study was to evaluate the role of hysteroscopic endometrial ablation in the treatment of menorrhagia.

Methods: two hundred patients were submitted to hysteroscopic endometrial ablation between April 1991 and April 1999. The surgery was performed in a private hospital under spinal anesthesia. In the first four years we used 1.5\% glycine as a distension medium and in the last four years we used mannitol 3\%. The follow-up ranged from 8 months to 8 years.

Results: relief of the symptomatology was achieved in 180 $(90 \%)$ patients. One episode of uterine perfuration, two cases of moderate liquid overload and five of hematometra were recorded. The histologic evaluation of the resected endometrium showed proliferative activity in 104 patients $(52 \%)$ and secretory activity in $34(17 \%)$. Endometrial hyperplasia was found in 20 patients $(10 \%)$. The patients submitted to resection showed a smaller uterine cavity and fibrous synechia, whereas in patients submitted to endometrial destruction the uterine cavity was small but without synechia. Two patients had moderade hypervolemia during the procedure, for which clinical treatment was successful.

Conclusions: Hysteroscopic endometrial ablation showed to be a safe and effective procedure as treatment of menorrhagia.

KEY WORDS: Disfunctional uterine bleeding. Endometrial hyperplasia. Hysteroscopic resection. Endometrial resection.

\section{Referências}

1. Cole P, Berlin I. Elective hysterectomy. Am J Obstet Gynecol 1977; 129:117-23.
2. Goldrath MH, Fuller TA, Segal S. Laser photovaporization of endometrium for the treatment of menorrhagia. Am J Obstet Gynecol 1981; 140:14-9.

3. DeCherney A, Polan ML. Hysteroscopic management of intrauterine lesions and intractable uterine bleeding. Obstet Gynecol 1983; 61:392-7.

4. Vancaillie TG. Electrocoagulation of the endometrium with the ball-end resectoscope. Obstet Gynecol 1989; 74:425-7.

5. Townsend DE, Richart RM, Paskowitz RA, Woolfork RE. Rollerball coagulation of the endometrium. Obstet Gynecol 1990; 76:310-3.

6. Amin HK, Neuwirth RS. Operative hysteroscopy utilizing dextran as a distending medium. Clin Obstet Gynecol 1983; 26:277-84.

7. Hamou J. Electroresection of fibroids. In: Sutton C, Diamond M, editors. Endoscopic Surgery for Gynaecologists. $1^{\text {st }}$ ed. London: W.B. Saunders; 1993. p.327.

8. Phipps JH, Lewis BV, Prior MV, Roberts T. Experimental and clinical studies with radiofrequency-induced thermal endometrial ablation for functional menorrhagia. Obstet Gynecol 1990; 76:876-81.

9. Neuwirth RS, Duran AA, Singer A, MacDonald R, Bolduc L. The endometrial ablator: a new instrument. Obstet Gynecol 1994; 83:792-6.

10. Magos AL, Baumann R, Lockwood GM, Turnbull AC. Experience with the first 250 endometrial resections for menorrhagia. Lancet 1991; 337:1074-8.

11. Brun G, Roussilhes R, Saurel J. Endométrectomie pour métrorragies: 45 cases. J Gynecol Obstet Biol Reprod (Paris) 1991; 20:532-7.

12. Hamou J. Hysteroscopy and Microcolpohysteroscopy. $1^{\text {st }}$ ed. Norwalk: Appleton \& Lange; 1991. p.186.

13. Maher PJ, Hill DJ. Transcervical endometrial resection for abnormal uterine bleeding: report of 100 cases and review of literature. Aust N Z J Obstet Gynaecol 1990; 30:357-60.

14. Petrucco OM, Gillespie A. The neodymium:YAG laser and the resectoscope for the treatment of menorrhagia. Med J Aust 1991; 154:518-20. 